\title{
Design and Acquisition of EOG Based Interactive Communications for ALS Patients
}

\author{
Neşe ÖZKAN*1, Ali IŞIK² ${ }^{2}$ Uğur FİDAN ${ }^{3}$
}

Accepted : 12/05/2017 Published: 21/08/2017

DOI: 10.18100/ijamec.2017Special Issue30460

\begin{abstract}
Amyotrophic lateral sclerosis (ALS) is a motor neuron disease caused by loss of function of spinal cord and brain stem nerve cells. Loss of function in nerve cells leads to weakness and explosion (atrophy) in the muscles. The outcome of the weakness in the muscles needs the help of someone. Despite the limitations of the movement, studies on methods of increasing the daily quality of life ALS patients are continuing. Electrooculogram (EOG) signals were taken from an instrumentation amplifier with $48 \mathrm{~dB}$ gain and $107 \mathrm{~dB}$ CMMR ratio. A $16 \mathrm{~Hz}$ Low Pass Filter and a $50 \mathrm{~Hz}$ Notch Filter were used to increase the signal to noise ratio. EOG signals are digitized with 10bit resolution ADC and applied to ATmega328 microcontroller. So, the software of microcontroller determines the horizontal and vertical movements of the eye. In this way, the interactive PC software was controlled by the EOG signals. EOG-based interactive software which was developed using the $\mathrm{C}$ \# programming language has provided to patients' daily requirements, social media accounts etc. The system has been tested on healthy subjects and it has been seen that people can control the software by eye movements. As a result, system will be useful not only for ALS patients, but also for permanent or partially bedridden patient (MS, Hemiplegia, etc.) groups. And also, while the quality of life in the patients' own habitats is being raised, they will also be able to benefit from health services within the scope of home health services.
\end{abstract}

Keywords: Amyotrophic Lateral Sclerosis (ALS), Atrophy, Electrooculogram (EOG), Home Health Care, Interactive

\section{Introduction}

ALS disease is a central nervous system disease caused by loss of function of motor cells (neurons) in the spinal cord and brain stem. Loss of function in the nerve cells leads to weakness and eruption (atrophy) in the muscles. This situation removes the movement functions of ALS patients and leaves them in need of someone's help. Although there are limitations of the movement, studies on the methods that increase the daily life quality of ALS patients who are not different from healthy individuals as consciousness continue.

In the literature, Barea et al. (2002) developed the humanmachine interface to control the wheelchair with EOG signals [1]. Kherlopian et al. (2006) developed a computer-based system capable of automatic analysis and processing of EOG signals. Signal processing techniques have been used to reduce noise in the EOG signal during work and to eliminate unwanted signals caused by muscle movements around the eye. The right, left, and center positions of the eye were determined by asking them to print on the screen by eye movements with 13 words and 5 words with 6 letters [2]. In another work, they created a computer keyboard interface and implemented a virtual keyboard that can be controlled with EOG and EMG marks. When the eye gaze is obtained with the EOG signal during operation, the user's muscle

\footnotetext{
1,2,3 Biomedical Engg., Afyon Kocatepe University, Afyonkarahisar03200, TURKEY

* Corresponding Author: Email: nozkan@aku.edu.tr

Note: This paper has been presented at the 5th International Conference on Advanced Technology \& Sciences (ICAT'17) held in Istanbul (Turkey), May 09-12, 2017.
}

movements are recorded with the EMG signal [3]. Bulling (2009) and the working group have designed EOG eyewear which provide daily environmental awareness and sensitivity. Real-time EOG signals can be received with the specially designed glasses and transmitted to the computer interface via Bluetooth. 8 games designed in the computer interface were requested to play with 11 eye movements and at the same time the EOG signals obtained were analyzed by signal processing techniques [4]. In 2012, Mingmin Yan and others developed a mouse-controlled system using EOG signals for ALS patients. The position of the eye was determined using signal processing techniques on the received EOG signals [5]. Ayvaz and Gürkan (2014) have designed an EOG circuit with 3 electrodes in order to control the actuators in their work [6]. In the other work they did in 2015, they developed the EOG based Human Computer Interface application. The system was tested on 10 people, the results of performing the desired eye movement commands were recorded and processed in MATLAB environment. [7-8]. Mondal et al (2015) have implemented EOG based prototype data system design. The signals from this low-cost EOG prototype demonstrated the success of the signal they received compared to the EOG module in the BIOPAC MP36 system [9]. In 2016, Tamura and others created EOG and EMG based computer interfaces. By applying signal processing techniques on the signals they have acquired with the interface they have created they have distinguished the right-left commands on the EOG sign [10]. In this study, it is aimed to realize a system that will enable EOG based interactive software to control many commands from daily necessities of ALS patients to social media accounts. In this way communication between the patient and the companion is ensured 
and interactive communication with the patient's social environment will be strengthened. While the quality of life is being upgraded within the patients' own living spaces, technological infrastructure will be provided for health services within the scope of home health services.

\section{Materials and Methods}

In general, home care services are offered at the professional level or by family members in the home or living environment of the individual, in order to maintain, raise and rehabilitate the health of the individual. 29280 dated February 27, 2015 by the Ministry of Health of the Republic of Turkey, "Health Services at Home by the Ministry of Health and its Affiliates" regulates the scope and content of home health services [11]. These services aim to protect the quality of life and the social dignity of the individual in a wide range of needs, including both health and social services. Home care services are generally expressed as services that take place in institutional care and reduce or delay the need to stay in institutions [12]. ALS and paralyzed patients are often in need of treatment within the scope of this regulation.

ALS disease is known to Aran Duchenne until 1840 and is known in many countries by the name Charcot. The first clinical and pathological description of ALS was made by Jean Martin Charcot (1874). In a case study of 25 patients, they defined ALS on the basis of various clinical findings such as weakness melting and pathological reflexes in the muscles [13]. ALS disease is a central nervous system disease caused by loss of function of motor cells (neurons) in the spinal cord and brain

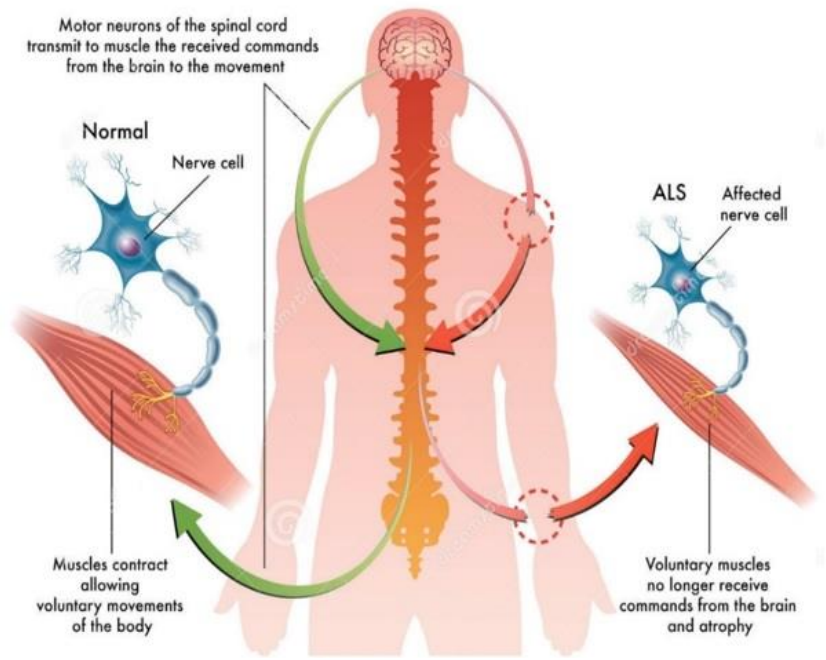

stem. Figure 1 shows how the muscular movements due to deformation in the nerve cells in ALS disease are restricted.

Fig. 1. Amyotrophic Lateral Sclerosis (ALS)

\subsection{Measurement of Electrooculogram (EOG) Signals}

Eye movements are provided by three pairs of muscles, mainly medial-lateral recti muscle, superior-inferior recti muscle and superior-inferior muscular muscle. The stimulus from the cerebral nerves induces potential tension in the ocular muscles with frequency components between $0.5 \mathrm{uV}$ and $0.3 \mathrm{~Hz}$ to $40 \mathrm{~Hz}$. These potential changes are detected by $\mathrm{Ag} / \mathrm{AgCl}$ electrodes and amplified by instrumentation amplifiers (Figure 2). To increase the $\mathrm{S} / \mathrm{N}$ ratio of the measuring system, the EOG signals are passed through the Notch and band pass filter.

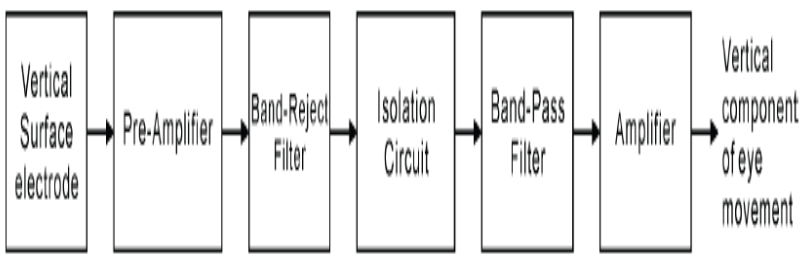

Fig. 2. EOG measuring circuit block diagram.

When the eyes move in different directions, the reciprocating eye muscles are stimulated. Thus, horizontal and vertical eye movements can be observed simultaneously. In Figure 3, EOG signs appear in the muscle groups surrounding the eye during vertical movements.

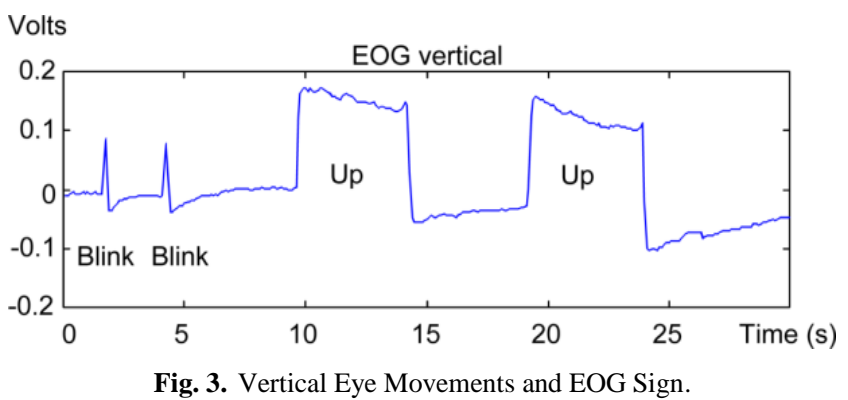

\subsection{EOG Based Interactive Communication System}

Figure 4 shows the block diagram of the EOG based interactive communication system design. The system consists of 3 basic parts. The first part forms the EOG measuring circuit, which translates eye movements into electrical action. It constitutes the second part of the digital process controller (DSP) system which converts from electrical signals to position information according to $360^{\circ}$ and converts the position information into mouse control signals and transfers the information to the PC via telemetry method. The last part is interactive PC software that will enable communication between the patient and the environment. Developed using the $\mathrm{C}$ \# programming language on the Visual Studio .NET platform, EOG based interactive software enables patients to control many commands from everyday needs to social media accounts.

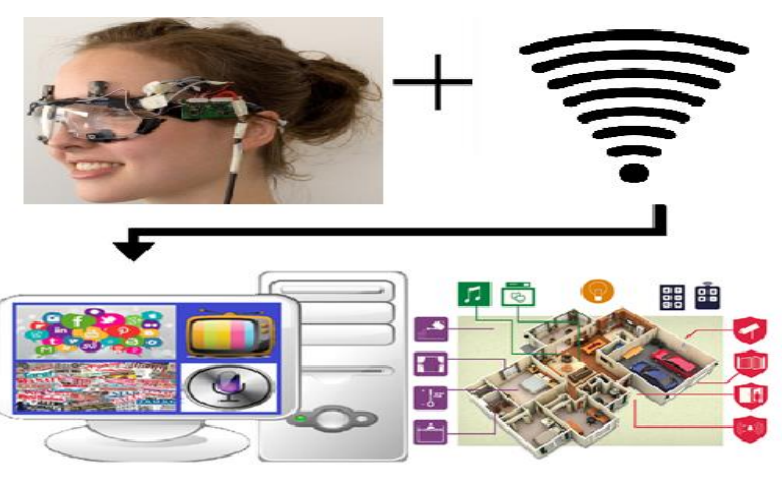

Fig. 4. EOG Based Interactive Communication System 


\section{Results}

In order to ergonomically place the EOG electrodes on the measurement surface, the eyewear frame was designed in the Solid Works program and was manufactured using the MassPortal Pharaoh ED model 3D printer (Fig. 5).

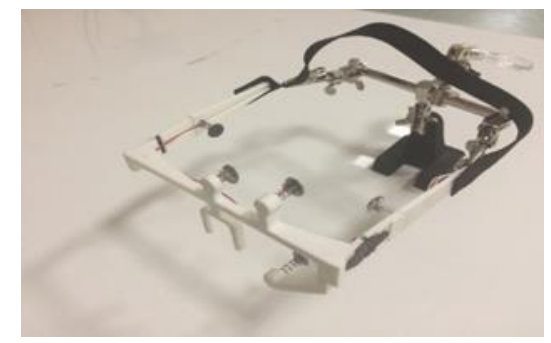

Fig. 5. Produced Eyewear model

The realized system open circuit diagram is shown in Figure 6. $\mathrm{Ag} / \mathrm{AgCl}$ electrodes were placed around the orbicularis oculi muscles. Electrooculogram (EOG) signals were taken from an instrumentation amplifier with $48 \mathrm{~dB}$ gain and $107 \mathrm{~dB}$ CMMR ratio. A $16 \mathrm{~Hz}$ Low Pass Filter and a $50 \mathrm{~Hz}$ Notch Filter were used to increase the signal to noise ratio. EOG signals are digitized with 10bit resolution ADC and applied to ATmega328 microcontroller. So, the software of microcontroller determines the transvers and sagittal movements of the eye. The movement information of the eye was sent to the computer with NRF24L01 transceiver at $2.4 \mathrm{GHz}$ operating frequency with $9.6 \mathrm{~Kb} / \mathrm{s}$ data transmission speed. This way, the interactive PC software was controlled by the EOG signals.

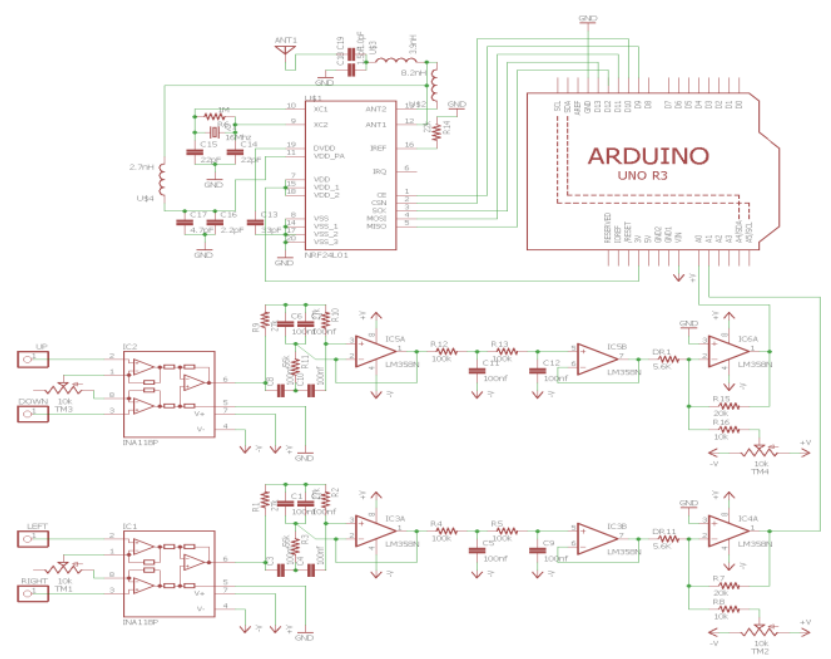

Fig. 6. Circuit diagram of the system

The Analog Discovery 2 data logger, manufactured by Digilent, was connected to both outputs of the EOG circuit to test that the hardware was tracking the movements of the eyeball. It is desirable for the person to make eye movements in accordance with the commands specified at a distance of about $80 \mathrm{~cm}$. Figure 7 shows the response of the eye sphere to the sagittal and transverse axis commands. During the eye blinking movements, there are $180^{\circ}$ phase different signals between the signals from both channels. The obtained data was analyzed by signal processing techniques and converted to a coordinate data to be detected by the monitor and transferred to the PC.

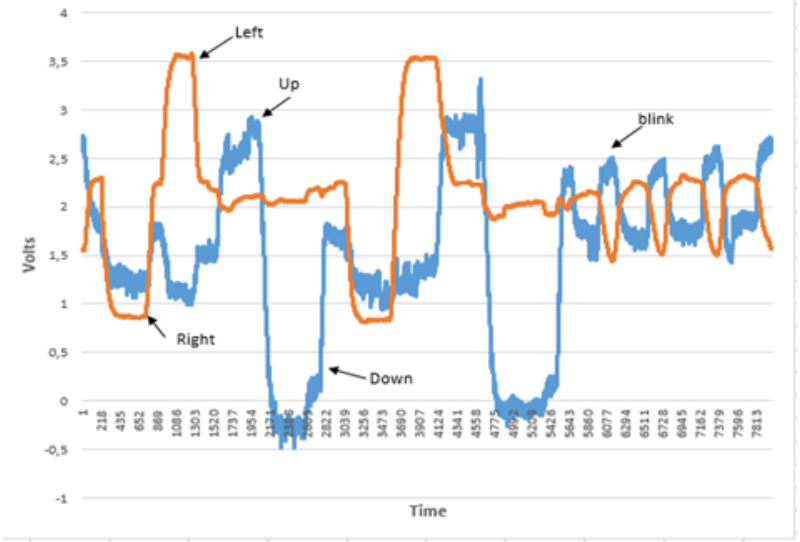

Fig. 7. Eye Movements

EOG-based interactive software developed using the $\mathrm{C}$ \# programming language The Lenovo Idea center model all in one is run on the Lenovo PC (Figure 8) with i5 processor with 21.5" $1920 * 1080$ resolution monitor capability. In order to enable interactive communication with the external environment of the patient, the software includes modules for Patient related, Conversation, Communication, Swift messages, Media and Social media. The distance between the monitor and the EOG measurement system was set to $80 \mathrm{~cm}$. It was desirable for the person to control the modules on the software with eye movements. Table 1 shows the functions to be controlled and the control periods. During the test, the person controlled all modules in the software with eye movements. The average duration of access to the modules is 3.24 seconds and the module selection time is 4.46 seconds.

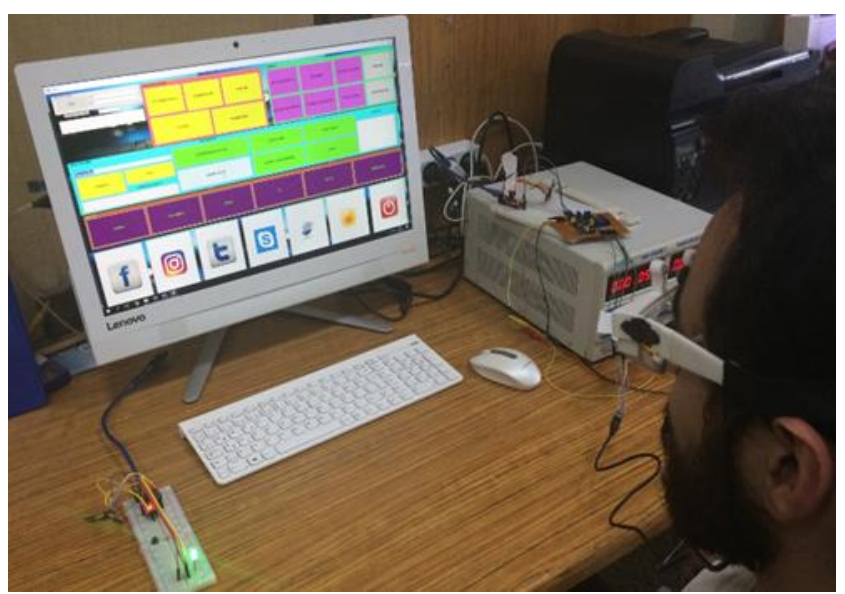

Fig. 8. Implemented Interface Program

Table 1. Accessing and Controlling Modules with EOG

\begin{tabular}{lcc} 
Modules & Arrival Time (s) & Departure Time (s) \\
Conversation & 6.16 & 8.18 \\
Communication & 0.84 & 3.03 \\
Swift messages & 2.87 & 3.14 \\
Media & 1.16 & 1.88 \\
Social media & 5.18 & 6.11 \\
\hline
\end{tabular}




\section{Discussion and Conclusions}

Amyotrophic Lateral Sclerosis (ALS) is a motor neuron disease which is caused by loss of function of the spinal cord and brain stem nerve cells. Loss of function in the nerve cells leads to weakness and eruption (atrophy) in the muscles. This situation eliminates the movement functions so patient needs to the assistance of a companion. Although there are limitations of the movement, studies on the methods that increase the daily life quality of ALS patients who are not different from healthy individuals as consciousness continue. In this study, the interactive PC software was controlled by the EOG signals. EOGbased interactive software which was developed using the $\mathrm{C} \#$ programming language has provided to patients' daily requirements, social media accounts etc. The system has been tested on healthy subjects and it has been seen that people can control the software by eye movements. As a result, system will be useful not only for ALS patients, but also for permanent or partially bedridden patient (MS, Hemiplegia, etc.) groups. And also, while the quality of life in the patients' own habitats is being raised, they will also be able to benefit from health services within the scope of home health services.

\section{Acknowledgements}

This study is supported by TÜBİTAK BİDEB Authority under the project numbered 2209/A program 1919B011601485. We would like to thank TUBITAK Scientific Human Resource Support Program for their support in conducting this research.

\section{References}

[1] R. Barea, L. Boquete, M. Mazo, E. López, "System for assisted mobility using eye movements based on electrooculography," IEEE Transactions on Neural Systems and Rehabilitation Engineering, 2002, 10(4), 209-218.

[2] A. R. Kherlopian, J. P. Gerrein, M. Yue, K. E. Kim, J.W. Kim, M. Sukumaran, P. Sajda, "Electrooculogram based system for computer control using a multiple feature classification model," In Engineering in Medicine and Biology Society, 2006, pp. 1295-1298.

[3] H. S. Dhillon, R. Singla, N. S. Rekhi, R. Jha, "EOG and EMG based virtual keyboard: A brain-computer interface," In Computer Science and Information Technology, 2009, pp. 259-262.

[4] A. Bulling, D. Roggen, G. Tröster, "Wearable EOG goggles: Seamless sensing and context-awareness in everyday environments," Journal of Ambient Intelligence and Smart Environments, 2009, 1(2), 157-171.

[5] M. Yan, H. Tamura, K. Tanno, "Development of mouse cursor control system using electrooculogram signals and its applications in revised hasegawa dementia scale task," In World Automation Congress (WAC), 2012, pp. 1-6.

[6] A. Ayvaz, G. Ertaş, "Design of an EOG Based Electronic Circuit to Control Actuators," National Congress of Medical Technology'14, Turkey, 2014, 22-25.

[7] S. Gürkan, P. A. Öner, A. İstanbullu, S. Doğan, "Designing a Portable Data Acquisition System for Human-Computer Interface Applications," National Congress of Medical Technology'15, Turkey, 2015, 165-168.

[8] S. Gürkan, G. Gürkan, A. Kaya, A. B. Uşaklı, “A Novel Eye-Blınk Based Easy Communication Device for Amyotrophic Lateral Sclerosis Patients: Eyepad®," 2015.

[9] C. Mondal, M. K. Azam, M. Ahmad, S.K. Hasan, M.R. Islam, "Design and implementation of a prototype electrooculography based data acquisition system," In Electrical Engineering and Information Communication Technology (ICEEICT), 2015 International Conference on, pp. 1-6.

[10] H. Tamura, M. Yan, K. Sakurai, K. Tanno, "EOG-sEMG Human Interface for Communication," Computational Intelligence and Neuroscience, 2016.

[11] Ministry of Health and its Affiliates Implementing Regulation On The Presentation of Home Health Care Services, 27.02.2015.
[12] M. Yılmaz, F. Sametoğlu, G. Akmeşe, A. Tak, B. Yağbasan, S. Gökçay, S. Erdem, "In-Home Health Services as an Alternative Form of Presentation of Patient Care,” Istanbul Med Journal, 2010.

[13] F. Koç and E. Tan, "Amyotrophic Lateral Sclerosis," TND Neuromuscular Diseases Scientific Working Group. 